\title{
On the dimension reduction in the quickest detection problem for diffusion processes with exponential penalty for the delay*
}

\author{
Bruno Buonaguidi ${ }^{\dagger}$
}

\begin{abstract}
The problem of the quickest detection of a change in the drift of a time-homogeneous diffusion process is considered under the assumption that the detection delay is exponentially penalized. In this framework, the past literature has shown that a twoor three-dimensional optimal stopping problem needs to be faced. In this note, we show how a change of measure significantly simplifies the setting by reducing the dimension of the optimal stopping problem to one or two, respectively. We illustrate this result in the well known Brownian motion case analyzed by Beibel [4] and when a Bessel process is observed, generalizing therefore the results for the linear penalty case obtained by Johnson and Peskir [13].
\end{abstract}

Keywords: Bessel process and Brownian motion; change-point/disorder problem; diffusion processes; dimension reduction; optimal stopping.

MSC2020 subject classifications: 60G40; 60J60; 62C10; 62L15.

Submitted to ECP on June 5, 2021, final version accepted on November 30, 2021.

\section{Introduction}

The quickest detection problem for a diffusion process $X:=\left(X_{t}\right)_{t \geq 0}$ aims at determining the moment $\theta$, known as disorder time or change-point, at which $X$ changes its drift. A good detection strategy should not raise an alarm too early to avoid false alarms and should be able to detect $\theta$ by making the detection delay as small as possible. In this work, we assume that $X$ is a time-homogeneous diffusion process and we approach the problem in the so called Bayesian setting with exponential penalty for the delay: $\theta$ takes on value 0 with positive probability and is exponentially distributed conditionally to the event $\theta>0$; the risk of our strategy is given by the linear combination between the probability of raising a false alarm and the exponential delay it takes to detect $\theta$ since its occurrence (see Section 2.1).

Problems of quickest detection for the drift of diffusion processes have been mostly studied under the linear penalty for the delay: [20] and [10] considered a Brownian motion within the infinite and finite horizon formulation, respectively; [13] studied the problem for a Bessel process; [12] focused on time-inhomogeneous Gaussian processes and fractional Brownian motion. The introduction of the exponential penalty is due

${ }^{*}$ Support from UCSC (D1 research grant) is also acknowledged.

${ }^{\dagger}$ Università Cattolica, Milan, Italy. E-mail: bruno. buonaguidi@unicatt.it 
to [18] who studied the discrete-time case for independent and identically distributed random variables; he motivated the exponential penalty for the delay as a more suitable measure to capture the true cost of a delayed action in some financial applications and in the health monitoring of components in interconnected systems. Later, [4] extended the Poor's Bayesian problem to the case of a Brownian motion and [11] generalized it to diffusion processes with non-constant drift. Problems of quickest detection with exponential penalty were also studied by [2], [3], [9] for simple and compound Poisson processes and by [6] for certain type of Lévy processes.

The results in [4] showed that, when the signal-to-noise ratio (defined as the difference between the new drift and the old drift divided by the diffusion coefficient) is constant, the initial quickest detection problem boils down to an optimal stopping problem for a two-dimensional Markov diffusion process, having as components the posterior probability $\Pi$ and the generalized odds $\Phi$ (defined in Section 2.1). The dimension further increases when the signal-to-noise ratio is not constant: in this situation, as shown in [11], the original problem can be reduced to an optimal stopping problem for the three-dimensional Markov diffusion process $(X, \Pi, \Phi)$, whose stochastic differential equations are expressed in terms of the innovation process. This makes the triplet $(X, \Pi, \Phi)$ strongly interdependent and, as a result, the analysis of the optimal stopping problem highly involved (see Section 2.2).

The main contribution of this note is thus twofold: (i) we show that a change of measure argument allows us to reduce the dimension of the optimal stopping problem from two to one, when the signal to-noise-ratio is constant, and from three to two, when it is not. In the first case, the optimal stopping problem is for the Markov diffusion process $\Phi$; in the second case, the optimal stopping problem is for the Markov diffusion process $(X, \Phi)$, whose stochastic differential equations are now expressed in terms of the original standard Brownian motion and get uncoupled in the first component. Accordingly, the problem becomes significantly simplified (see Section 3). (ii) Under this new setting, we obtain the solution for the prominent case of the Brownian motion, thus providing an alternative approach to that in [4] (see Section 4). Then, we solve the problem for a Bessel process, which is characterized by a non-constant signal-to-noise ratio; to the best of our knowledge, this is the first time that the quickest detection problem with exponential penalty is formulated for such a process (see Section 5). Recalling that the exponential penalty contains the linear penalty as a limit case (see, e.g., [4, Remark 2] and [2]), our results extend those obtained in [13], where the the linear penalty was studied.

\section{Formulation of the problem}

In this section we describe the problem of the quickest detection with exponential penalty for the drift of a time-homogeneous diffusion process following the lines of arguments in [11].

\subsection{Description of the problem}

On the probability space $\left(\Omega, \mathscr{F}, \mathbb{P}_{\pi}\right)$ a standard Brownian motion $W:=\left(W_{t}\right)_{t \geq 0}$ and a non-negative random variable $\theta$ are defined, with $W$ and $\theta$ being independent. The probability measure $\mathbb{P}_{\pi}$ is defined by

$$
\mathbb{P}_{\pi}:=\pi \mathbb{P}^{0}+(1-\pi) \int_{0}^{\infty} \mathbb{P}^{s} \lambda e^{-\lambda s} d s
$$

where $\mathbb{P}^{s}$ is the probability measure under which $\mathbb{P}^{s}(\theta=s)=1, s \geq 0$, and $\pi \in[0,1)$ and $\lambda>0$ are two given and fixed values. From (2.1) it follows that $\mathbb{P}_{\pi}(\theta=0)=\pi$ and 
Dimension reduction in the quickest detection problem with exponential penalty

$\mathbb{P}_{\pi}(\theta>t \mid \theta>0)=e^{-\lambda t}$, for $t>0$. The same space hosts a time-homogeneous diffusion process, which solves the stochastic differential equation

$$
d X_{t}=\left(\mu_{0}\left(X_{t}\right)+\mathbb{1}_{\{t \geq \theta\}}\left(\mu_{1}\left(X_{t}\right)-\mu_{0}\left(X_{t}\right)\right)\right) d t+\sigma\left(X_{t}\right) d W_{t}, \quad X_{0}=x \in \mathbb{S}
$$

where $\mathbb{S}$ is the state space of $X, \sigma(\cdot)>0$ is the diffusion coefficient, while $\mu_{0}(\cdot)$ and $\mu_{1}(\cdot)$ are the drift of $X$ before and after the occurrence of $\theta$, respectively. The latter is therefore called disorder time or change-point, after which $X$ changes its drift.

Through the sequential monitoring of $X$ only, the aim is to determine a stopping time of $X$ at which we can optimally interrupt the observation and raise an alarm. More precisely, solving the Bayesian quickest detection problem with exponential penalty for the delay means computing the value function

$$
V(\pi):=\inf _{\tau}\left(\mathbb{P}_{\pi}(\tau<\theta)+c \mathbb{E}_{\pi}\left[e^{\alpha(\tau-\theta)^{+}}-1\right]\right), \quad \pi \in[0,1),
$$

and determining the optimal stopping time $\tau^{\star}$ at which the infimum (taken over all the stopping times $\tau$ of $X)$ is attained. In the expression above, $\mathbb{P}_{\pi}(\tau<\theta)$ is the probability of a false alarm and $\mathbb{E}_{\pi}\left[e^{\alpha(\tau-\theta)^{+}}-1\right]$ is the expected exponential detection delay associated with a stopping time $\tau$ of $X ; \alpha>0$ is the known value at which the losses due to the detection delay are compounded and $c>0$ is a given value which weights their relevance.

The analysis of (2.3) requires the introduction of the following quantities depending on $X$ :

$$
L_{t}:=\frac{d \mathbb{P}_{t}^{0}}{d \mathbb{P}_{t}^{\infty}}, \quad \pi_{t}:=\mathbb{P}_{\pi}\left(\theta \leq t \mid \mathscr{F}_{t}^{X}\right), \quad \Phi_{t}:=\frac{\mathbb{E}_{\pi}\left[e^{\alpha(t-\theta)} \mathbb{1}_{\{\theta \leq t\}} \mid \mathscr{F}_{t}^{X}\right]}{1-\pi_{t}},
$$

where $L:=\left(L_{t}\right)_{t \geq 0}$ is the likelihood ratio process, $\Pi:=\left(\pi_{t}\right)_{t \geq 0}$ is the posterior probability process and $\Phi:=\left(\Phi_{t}\right)_{t \geq 0}$ is the generalized odds process. In the expressions above, $\mathscr{F}_{t}^{X}$ is the sigma-algebra generated by $X$ up to $t \geq 0, \mathbb{P}^{\infty}$ is the probability measure under which the drift of $X$ never changes (i.e., $\mathbb{P}^{\infty}(\theta=\infty)=1$ ), and $\mathbb{P}_{t}^{0}$ and $\mathbb{P}_{t}^{\infty}$ are the restrictions of $\mathbb{P}^{0}$ and $\mathbb{P}^{\infty}$ to $\mathscr{F}_{t}^{X}$. Girsanov's theorem and the results in [2] also show that $L$ and $\Phi$ admits the expressions

$$
\begin{aligned}
L_{t} & =\exp \left(\int_{0}^{t} \frac{\mu_{1}\left(X_{s}\right)-\mu_{0}\left(X_{s}\right)}{\sigma^{2}\left(X_{s}\right)} d X_{s}-\frac{1}{2} \int_{0}^{t} \frac{\mu_{1}^{2}\left(X_{s}\right)-\mu_{0}^{2}\left(X_{s}\right)}{\sigma^{2}\left(X_{s}\right)} d s\right), \\
\Phi_{t} & =e^{(\alpha+\lambda) t} L_{t}\left(\Phi_{0}+\lambda \int_{0}^{t} \frac{e^{-(\alpha+\lambda) s}}{L_{s}} d s\right)
\end{aligned}
$$

which will be used in Sections 4 and 5 to disclose the explicit structure of the optimal detection strategy.

\subsection{A three-dimensional optimal stopping problem}

In [11] it was shown that $X, \Pi$, and $\Phi$ evolve according to the stochastic differential equations

$$
\begin{aligned}
d X_{t} & =\left(\left(1-\pi_{t}\right) \mu_{0}\left(X_{t}\right)+\pi_{t} \mu_{1}\left(X_{t}\right)\right) d t+\sigma\left(X_{t}\right) d \tilde{W}_{t}, \quad X_{0}=x \\
d \pi_{t} & =\lambda\left(1-\pi_{t}\right) d t+\rho\left(X_{t}\right) \pi_{t}\left(1-\pi_{t}\right) d \tilde{W}_{t}, \quad \pi_{0}=\pi \\
d \Phi_{t} & =\left(\lambda+(\lambda+\alpha) \Phi_{t}+\rho^{2}\left(X_{t}\right) \pi_{t} \Phi_{t}\right) d t+\rho\left(X_{t}\right) \Phi_{t} d \tilde{W}_{t}, \quad \Phi_{0}=\frac{\pi}{1-\pi}
\end{aligned}
$$

where $\rho(\cdot)$ is the so called signal-to-noise ratio, given by

$$
\rho(\cdot):=\frac{\mu_{1}(\cdot)-\mu_{0}(\cdot)}{\sigma(\cdot)},
$$


and $\tilde{W}:=\left(\tilde{W}_{t}\right)_{t \geq 0}$, defined by

$$
\tilde{W}_{t}:=\int_{0}^{t} \frac{d X_{s}}{\sigma\left(X_{s}\right)}-\int_{0}^{t} \frac{\left(1-\pi_{s}\right) \mu_{0}\left(X_{s}\right)+\pi_{s} \mu_{1}\left(X_{s}\right)}{\sigma\left(X_{s}\right)} d s
$$

is the innovation process/standard Brownian motion under $\mathbb{P}_{\pi}$ with respect to the filtration $\mathscr{F}^{X}$.

The value function $V$ from (2.3) was also reduced to the equivalent optimal stopping problem

$$
V(\pi)=\inf _{\tau} \mathbb{E}_{\pi}\left[1-\pi_{\tau}^{\pi}+c \alpha \int_{0}^{\tau}\left(1-\pi_{t}^{\pi}\right) \Phi_{t}^{\frac{\pi}{1-\pi}} d t\right],
$$

where the infimum is taken over all the stopping times $\tau$ of $X$ and the superscripts indicate the dependence of the processes $\Pi$ and $\Phi$ on their starting points. When $\rho$ is constant, $(\Pi, \Phi)$ from (2.8)-(2.9) is a strong Markov process and, to approach (2.12), $(\Pi, \Phi)$ must be enabled to start at any point $(\pi, \phi) \in[0,1) \times[0, \infty)$ under the probability measure $\mathbb{P}_{\pi, \phi}$, under which $\left(\pi_{0}, \Phi_{0}\right)=(\pi, \phi)$. In this way, one needs to consider the extended two-dimensional optimal stopping problem

$$
V(\pi, \phi)=\inf _{\tau} \mathbb{E}_{\pi, \phi}\left[1-\pi_{\tau}+c \alpha \int_{0}^{\tau}\left(1-\pi_{t}\right) \Phi_{t} d t\right]
$$

where the infimum is taken over all the stopping times $\tau$ of $(\Pi, \Phi)$. Moreover, when $\rho$ is not constant, only the triplet $(X, \Pi, \Phi)$ from (2.7)-(2.9) is strongly Markovian and, to tackle (2.12), $(X, \Pi, \Phi)$ must be enabled to start at any point $(x, \pi, \phi) \in \mathbb{S} \times[0,1) \times[0, \infty)$ under the probability measure $\mathbb{P}_{x, \pi, \phi}$, under which $\left(X_{0}, \pi_{0}, \Phi_{0}\right)=(x, \pi, \phi)$. In this case, one ends up with the extended three-dimensional optimal stopping problem

$$
V(x, \pi, \phi)=\inf _{\tau} \mathbb{E}_{x, \pi, \phi}\left[1-\pi_{\tau}+c \alpha \int_{0}^{\tau}\left(1-\pi_{t}\right) \Phi_{t} d t\right],
$$

where the infimum is taken over all the stopping times $\tau$ of $(X, \Pi, \Phi)$. Sufficient conditions for the existence of a solution to (2.14) were provided in [11, Section 3].

\section{Reduction to an optimal stopping problem of lower dimension}

The arguments in [11] are based on the system of stochastic differential equations (2.7)-(2.9) under the probability measure $\mathbb{P}_{\pi}$; the fact that $X, \Pi$ and $\Phi$ are strongly interdependent and the high dimensionality of the optimal stopping problem (2.14) make its analysis very complex. Now we see that a change of measure allows us to move from the optimal stopping problem (2.14) for $(X, \Pi, \Phi)$ to an optimal stopping problem for $(X, \Phi)$, whose stochastic differential equations get uncoupled in $X$. These facts simplify considerably the analysis of the problems analyzed in the subsequent sections.

The next proposition derives the stochastic differential equations of $X, \Pi$, and $\Phi$ under $\mathbb{P}^{\infty}$.

Proposition 3.1. Under $\mathbb{P}^{\infty}, X, \Phi$ and $\Pi$ solve the stochastic differential equations

$$
\begin{aligned}
& d X_{t}=\mu_{0}\left(X_{t}\right) d t+\sigma\left(X_{t}\right) d W_{t}, \quad X_{0}=x, \\
& d \Phi_{t}=\left(\lambda+(\lambda+\alpha) \Phi_{t}\right) d t+\rho\left(X_{t}\right) \Phi_{t} d W_{t}, \quad \Phi_{0}=\frac{\pi}{1-\pi}, \\
& d \pi_{t}=\left(\lambda\left(1-\pi_{t}\right)-\rho^{2}\left(X_{t}\right) \pi_{t}^{2}\left(1-\pi_{t}\right)\right) d t+\rho\left(X_{t}\right) \pi_{t}\left(1-\pi_{t}\right) d W_{t}, \quad \pi_{0}=\pi,
\end{aligned}
$$

with $(X, \Phi)$ being a strong Markov diffusion process. 
Dimension reduction in the quickest detection problem with exponential penalty

Proof. Equation (3.1) is obtained from (2.2) by setting $\theta=\infty$. Then, replacing (3.1) in (2.11), we have

$$
d \tilde{W}_{t}=d W_{t}-\pi_{t} \rho\left(X_{t}\right) d t .
$$

By substituting now (3.4) in (2.8) and (2.9) we obtain (3.2) and (3.3). The fact that $(X, \Phi)$ is strongly Markovian comes from [14, Theorem 7.2.4].

Now we are going to show that the optimal stopping problem (2.12) admits an equivalent formulation under the measure $\mathbb{P}^{\infty}$. Denoting by $\mathbb{P}_{\tau}^{\infty}$ and $\mathbb{P}_{\pi, \tau}$ the restrictions of the measures $\mathbb{P}^{\infty}$ and $\mathbb{P}_{\pi}$ to $\mathscr{F}_{\tau}^{X}$, let us recall from [13, Lemma 1] that the RadonNikodym derivative of $\mathbb{P}_{\pi, \tau}$ with respect to $\mathbb{P}_{\tau}^{\infty}$ is given by

$$
\frac{d \mathbb{P}_{\pi, \tau}}{d \mathbb{P}_{\tau}^{\infty}}=e^{-\lambda \tau} \frac{1-\pi}{1-\pi_{\tau}},
$$

for all stopping times $\tau$ of $X$. Recalling that $\Phi$ starts at $\frac{\pi}{1-\pi}$ and that this dependence will be indicated by a superscript to $\Phi$ when needed, we can state the following theorem.

Theorem 3.2. The value function (2.12) satisfies the identity

$$
V(\pi)=(1-\pi)(1+c \alpha \hat{V}(\pi)),
$$

where

$$
\hat{V}(\pi):=\inf _{\tau} \mathbb{E}^{\infty}\left[\int_{0}^{\tau} e^{-\lambda t}\left(\Phi_{t}^{\frac{\pi}{1-\pi}}-\frac{\lambda}{c \alpha}\right) d t\right],
$$

for $\pi \in[0,1)$, with the infimum taken over all the stopping times $\tau$ of $X$.

Proof. By standard localization arguments and the application of the monotone and dominated convergence theorems, it is enough to show that (3.6)-(3.7) hold true for all bounded stopping times $\tau$ of $X$ such that $\left(\Phi_{t \wedge \tau}\right)_{t \geq 0}$ and $\left(\rho\left(X_{t \wedge \tau}\right)\right)_{t>0}$ are bounded. Let such a stopping time be given and fixed in the sequel. Recalling that $\Pi$ and $\Phi$ solve (2.8) and (2.9) under $\mathbb{P}_{\pi}$, it follows that

$$
1-\pi_{t}=1-\pi-\lambda \int_{0}^{t}\left(1-\pi_{s}\right) d s-\int_{0}^{t} \rho\left(X_{s}\right) \pi_{s}\left(1-\pi_{s}\right) d \tilde{W}_{s} .
$$

Then, applying Itô's product formula to $\left(\left(1-\pi_{t}\right) \Phi_{t}\right)_{t \geq 0}$ we have

$$
\left(1-\pi_{t}\right) \Phi_{t}=\pi+\int_{0}^{t}\left(1-\pi_{s}\right)\left(\lambda+\alpha \Phi_{s}\right) d s+\int_{0}^{t}\left(1-\pi_{s}\right)^{2} \rho\left(X_{s}\right) \Phi_{s} d \tilde{W}_{s} .
$$

Using the definition of $\tau$ and the fact that the two stochastic integrals in (3.8)-(3.9) are local martingales, according to the optional sampling theorem from (3.8) we have

$$
\mathbb{E}_{\pi}\left[\lambda \int_{0}^{\tau}\left(1-\pi_{t}\right) d t\right]=\mathbb{E}_{\pi}\left[\pi_{\tau}\right]-\pi,
$$

and from (3.9)-(3.10) we obtain

$$
\begin{aligned}
\mathbb{E}_{\pi}\left[\int_{0}^{\tau}\left(1-\pi_{t}\right) \Phi_{t} d t\right] & =\frac{1}{\alpha}\left(-\pi+\mathbb{E}_{\pi}\left[\left(1-\pi_{\tau}\right) \Phi_{\tau}\right]-\mathbb{E}_{\pi}\left[\lambda \int_{0}^{\tau}\left(1-\pi_{t}\right) d t\right]\right) \\
& =\frac{1}{\alpha} \mathbb{E}_{\pi}\left[\left(1-\pi_{\tau}\right) \Phi_{\tau}-\pi_{\tau}\right] .
\end{aligned}
$$

Then, from (2.12) and (3.11) we have

$$
\begin{aligned}
\mathbb{E}_{\pi}\left[1-\pi_{\tau}+c \alpha \int_{0}^{\tau}\left(1-\pi_{t}\right) \Phi_{t} d t\right] & =\mathbb{E}_{\pi}\left[1-\pi_{\tau}+c\left(\left(1-\pi_{\tau}\right) \Phi_{\tau}-\pi_{\tau}\right)\right] \\
& =(1-\pi) \mathbb{E}^{\infty}\left[e^{-\lambda \tau}+c e^{-\lambda \tau}\left(\Phi_{\tau}-\frac{\pi_{\tau}}{1-\pi_{\tau}}\right)\right],
\end{aligned}
$$


Dimension reduction in the quickest detection problem with exponential penalty

where the second equality is based on the change of measure (3.5).

Motivated by the structure of (3.12), let $\varphi_{t}:=\frac{\pi_{t}}{1-\pi_{t}}$ for $t \geq 0$. Then, by means of (3.3) and the application of Itô's formula to $\left(\varphi_{t}\right)_{t \geq 0}$ and $\left(e^{-\lambda t}\left(\Phi_{t}-\varphi_{t}\right)\right)_{t \geq 0}$ we have

$$
\begin{gathered}
\varphi_{t}=\frac{\pi}{1-\pi}+\lambda \int_{0}^{t}\left(1+\varphi_{s}\right) d s+\int_{0}^{t} \rho\left(X_{s}\right) \varphi_{s} d W_{s} \\
e^{-\lambda t}\left(\Phi_{t}-\varphi_{t}\right)=\alpha \int_{0}^{t} e^{-\lambda s} \Phi_{s} d s+\int_{0}^{t} e^{-\lambda s} \rho\left(X_{s}\right)\left(\Phi_{s}-\varphi_{s}\right) d W_{s} .
\end{gathered}
$$

According to the definition of $\tau$, the fact that the stochastic integral in (3.14) is a local martingale under $\mathbb{P}^{\infty}$ and the application of the optional sampling theorem, we have

$$
\mathbb{E}^{\infty}\left[e^{-\lambda \tau}\left(\Phi_{\tau}-\varphi_{\tau}\right)\right]=\alpha \mathbb{E}^{\infty}\left[\int_{0}^{\tau} e^{-\lambda t} \Phi_{t} d t\right]
$$

Finally, from (3.12), (3.15) and the equality $e^{-\lambda \tau}=1-\lambda \int_{0}^{\tau} e^{-\lambda t} d t$, it follows that

$$
\mathbb{E}_{\pi}\left[1-\pi_{\tau}+c \alpha \int_{0}^{\tau}\left(1-\pi_{t}\right) \Phi_{t} d t\right]=(1-\pi)\left(1+c \alpha \mathbb{E}^{\infty}\left[\int_{0}^{\tau} e^{-\lambda t}\left(\Phi_{t}^{\frac{\pi}{1-\pi}}-\frac{\lambda}{c \alpha}\right) d t\right]\right),
$$

which completes the proof.

To approach the optimal stopping problem (3.7) for the strong Markov process $(X, \Phi)$ we enable it to start at any point $(x, \phi) \in \mathbb{S} \times[0, \infty)$, so that (3.7) can be extended to

$$
\hat{V}(x, \phi):=\inf _{\tau} \mathbb{E}_{x, \phi}^{\infty}\left[\int_{0}^{\tau} e^{-\lambda t}\left(\Phi_{t}-\frac{\lambda}{c \alpha}\right) d t\right],
$$

where the expectation is with respect to the probability measure $\mathbb{P}_{x, \phi}^{\infty}$, under which $\left(X_{0}, \Phi_{0}\right)=(x, \phi)$, and the infimum is taken over all the stopping times $\tau$ of $(X, \Phi)$. In this way we have reduced the three-dimensional optimal stopping problem (2.14) to a twodimensional optimal stopping problem for the strong Markov process $(X, \Phi)$ satisfying (3.1)-(3.2), whose first component is uncoupled from the second one.

Let us observe that the optimal stopping problem (3.16) is Lagrange formulated; then, consistently to [16, Chapter 1], we may affirm that its continuation and stopping sets are defined by

$$
\begin{aligned}
& C:=\{(x, \phi) \in \mathbb{S} \times[0, \infty): \hat{V}(x, \phi)<0\}, \\
& D:=\{(x, \phi) \in \mathbb{S} \times[0, \infty): \hat{V}(x, \phi)=0\} .
\end{aligned}
$$

Noting that the integrand in (3.16) is negative when $\Phi_{t}<\lambda /(c \alpha)$, we conclude that $\{(x, \phi): \phi<\lambda /(c \alpha)\} \subset C$; equivalently, we can say that the least boundary between $C$ and $D$, defined by

$$
b(x):=\inf \{\phi \in[0, \infty):(x, \phi) \in D\}, \quad x \in \mathbb{S},
$$

satisfies $b(x) \geq \lambda /(c \alpha)$, for all $x \in \mathbb{S}$. It is also immediate to notice that the value function $\hat{V}$ is bounded and satisfies $-(c \alpha)^{-1} \leq \hat{V} \leq 0$, where the first inequality is due to the fact that $\phi-\lambda /(c \alpha) \geq-\lambda /(c \alpha)$ for $\phi \geq 0$, while the second equality comes from the fact that $\tau=0$ is an admissible stopping time and the definition of $\hat{V}$.

We conclude this section by deriving the infinitesimal generator $\mathbb{L}_{(X, \Phi)}$ of $(X, \Phi)$ under $\mathbb{P}^{\infty}$, which will be exploited in the next sections to construct the free-boundary 
problem that the value function $\hat{V}$ and the free-boundary $b$ solve. Standard arguments based on the application of Itô's formula imply that

$$
\mathbb{L}_{(X, \Phi)}=(\lambda+(\lambda+\alpha) \phi) \frac{\partial}{\partial \phi}+\mu_{0}(x) \frac{\partial}{\partial x}+\phi \rho(x) \sigma(x) \frac{\partial^{2}}{\partial \phi \partial x}+\frac{1}{2} \phi^{2} \rho^{2}(x) \frac{\partial^{2}}{\partial \phi^{2}}+\frac{1}{2} \sigma^{2}(x) \frac{\partial^{2}}{\partial x^{2}} .
$$

Denoted by $C^{i}(\cdot)$ the class of $i$-times continuously differentiable functions on the specified set ., $\mathbb{L}_{(X, \Phi)}$ acts on $f \in C^{2}(\mathbb{S} \times[0, \infty))$; we also observe that $\mathbb{L}_{(X, \Phi)}$ is of parabolic type (see, e.g. [15, Section 2]) and this is a consequence of the fact that $X$ and $\Phi$ are driven by the same original/standard Brownian motion $W$ under $\mathbb{P}^{\infty}$, as evident from (3.1)-(3.2).

Remark 3.3. The change of measure from $\mathbb{P}_{\pi}$ to $\mathbb{P}^{\infty}$ in the linear penalty framework analyzed in [13] has the effect to move from a two-dimensional optimal stopping problem for the process $(X, \Pi)$ solving (2.7)-(2.8) to another two-dimensional optimal stopping problem for the process $(X, \Phi)$ solving (3.1)-(3.2), with $\alpha=0$ in the latter equation, without leading to any dimensionality simplification. This is a substantial difference with respect to the logic behind our result, whose primary aim is the dimension shrinkage of the problem, while retaining the effect of decoupling the two-dimensional sufficient statistic $(X, \Phi)$ from (3.1)-(3.2) in $X$.

\section{Quickest detection for a Brownian motion}

In this section we solve the quickest detection problem (2.3) when $X$ is a Brownian motion changing its drift from one value to another. Then, (2.2) holds with $\mu_{0}, \mu_{1}, \sigma$ being constant values. This problem was studied under the probability measure $\mathbb{P}_{\pi}$ in [4], who treated the optimal stopping problem (2.12) as a generalized parking problem, using an approach similar to [5]. In this case $\rho$ from (2.10) is constant and, therefore, (2.12) is a two-dimensional optimal stopping problem for the process $(\Pi, \Phi)$ leading to (2.13).

However, Theorem 3.2, the same arguments used in the last part of Section 3 and the fact that $\rho$ is constant show that we can equivalently focus on the one-dimensional optimal stopping problem

$$
\hat{V}(\phi):=\inf _{\tau} \mathbb{E}_{\phi}^{\infty}\left[\int_{0}^{\tau} e^{-\lambda t}\left(\Phi_{t}-\frac{\lambda}{c \alpha}\right) d t\right], \quad \phi \in[0, \infty),
$$

for the strong Markov process $\Phi$, which solves (3.2). The expectation in (4.1) is with respect to $\mathbb{P}_{\phi}^{\infty}$, under which $\Phi_{0}=\phi$, and the infimum is taken over all the stopping times $\tau$ of $\Phi$. The continuation and stopping sets from (3.17)-(3.18) reduce to

$$
C=\{\phi \in[0, \infty): \hat{V}(\phi)<0\}, \quad D=\{\phi \in[0, \infty): \hat{V}(\phi)=0\},
$$

with the boundary $b$ from (3.19) being now constant. Then, the general theory of optimal stopping for strong Markov processes (see, e.g., [16, Chapter 3 and 4] or [20, Chapter 3]) leads us to formulate the following free-boundary problem for $\hat{V}$ and $b$ :

$$
\begin{aligned}
& \left(\mathbb{L}_{\Phi} \hat{V}-\lambda \hat{V}\right)(\phi)=-\left(\phi-\frac{\lambda}{c \alpha}\right), \quad \phi \in C=[0, b), \\
& \hat{V}(\phi)=0, \quad \phi \in D=[b, \infty) \quad \text { (instantaneous stopping), } \\
& \hat{V}^{\prime}(b)=0 \quad \text { (smooth fit), }
\end{aligned}
$$

where $\mathbb{L}_{\Phi}$ is the infinitesimal generator of $\Phi$ and is given by

$$
\mathbb{L}_{\Phi}=(\lambda+(\lambda+\alpha) \phi) \frac{\partial}{\partial \phi}+\frac{1}{2} \rho^{2} \phi^{2} \frac{\partial^{2}}{\partial \phi^{2}},
$$


which acts on $f \in C^{2}([0, \infty))$ and follows by discarding in (3.20) the addends whose differential operator depends on $x$. Let us define

$$
k_{1}:=\frac{2(\lambda+\alpha)}{\rho^{2}}, \quad k_{2}:=\frac{2 \lambda}{\rho^{2}}, \quad k_{3}:=\frac{k_{1}-1+\sqrt{\left(1-k_{1}\right)^{2}+4 k_{2}}}{2},
$$

and let $U(a, b, z)$ be the Kummer function

$$
U(a, b, z):=\frac{1}{\Gamma(a)} \int_{0}^{\infty} e^{-z t} t^{a-1}(1+t)^{b-a-1} d t,
$$

where $\Gamma(a):=\int_{0}^{\infty} x^{a-1} e^{-x} d x$ (see, e.g., [1, Eq. (13.2.5)]). Then, solution methods for degenerate hypergeometric equations (see [17, Cases 2.1.2.129 and 2.1.2.103]), as well as the conditions (4.4)-(4.5), show that the function $\hat{V}$ solving (4.3)-(4.5) is given by

$$
\hat{V}(\phi)=\left\{\begin{array}{l}
h g(\phi)-\frac{\phi}{\alpha}-\frac{1+c}{c \alpha}, \quad 0 \leq \phi<b, \\
0, \quad \phi \geq b
\end{array}\right.
$$

where the function $g($.$) and the constant h$ are given by

$$
g(\phi):=\left(\frac{1}{\phi}\right)^{k_{3}} U\left(k_{3}, 2 k_{3}+2-k_{1}, \frac{k_{2}}{\phi}\right), \quad h:=\frac{1+c(1+b)}{c \alpha g(b)},
$$

with the boundary point $b$ which solves uniquely

$$
\left(b+\frac{1+c}{c}\right) \frac{g^{\prime}(b)}{g(b)}-1=0 .
$$

Applying then the Itô-Tanaka formula to $\hat{V}$ from (4.9), which boils down to Itô's formula due to the smooth fit (4.5), and making use of the optional sampling theorem, it is easily verified that the solution to the free-boundary problem (4.3)-(4.5) coincides with that of the optimal stopping problem (4.1) and that the optimal stopping time $\tau^{\star}$ for the latter is given by

$$
\tau^{\star}=\inf \left\{t \geq 0: \Phi_{t} \geq b\right\} .
$$

In (4.12) $\Phi_{t}$ takes the more explicit form

$$
\Phi_{t}=e^{(\alpha+\lambda) t+\frac{\rho}{\sigma}\left(X_{t}-\frac{1}{2}\left(\mu_{1}+\mu_{0}\right) t\right)}\left(\frac{\pi}{1-\pi}+\lambda \int_{0}^{t} e^{-(\alpha+\lambda) s-\frac{\rho}{\sigma}\left(X_{s}-\frac{1}{2}\left(\mu_{1}+\mu_{0}\right) s\right)} d s\right),
$$

which arises from (2.5)-(2.6), upon recalling that $\mu_{0}, \mu_{1}, \sigma$ and $\rho$ are constant. Finally, we have that the value function $V$ of the initial problem (2.3) and (2.12) is given by

$$
V(\pi)=(1-\pi)\left(1+c \alpha \hat{V}\left(\frac{\pi}{1-\pi}\right)\right), \quad \pi \in[0,1),
$$

which follows from (3.6)-(3.7) in Theorem 3.2.

Remark 4.1. Alternatively to the free-boundary approach, optimal stopping problems for $\Phi$ when $\rho$ is constant could also be faced by resorting to the theory of optimal stopping for one-dimensional diffusions (see, e.g., [7], [8], [19]), with the latter having the advantage that there is no need to guess the structure of the optimal stopping set. 
Dimension reduction in the quickest detection problem with exponential penalty

\section{Quickest detection for a Bessel process}

In this section we study the quickest detection problem (2.3) when $X$ is a Bessel process changing its dimension from $\delta_{0} \geq 2$ to $\delta_{1}>\delta_{0}$ at time $\theta$. Then, $X$ evolves according to (2.2) with

$$
\mu_{0}(x)=\frac{\delta_{0}-1}{2 x}, \quad \mu_{1}(x)=\frac{\delta_{1}-1}{2 x}, \text { with } \delta_{1}>\delta_{0} \geq 2, \quad \text { and } \quad \sigma(x)=1, \quad x>0 .
$$

This problem has an appealing interpretation when $\delta_{0}=2$ and $\delta_{1}=3$, because it represents the situation where, through the sequential monitoring of the distance that a Brownian particle has from the origin, we want to detect the moment at which the particle moves from the two-dimensional plane to the three-dimensional space. The formulation of this problem is due to [13], where a linear penalty for the detection delay was adopted. As we said in Section 1, the exponential penalty considered in the present paper generalizes the linear penalty.

From (2.10) and (5.1) we observe that the signal-to-noise ratio takes the form

$$
\rho(x)=\frac{\gamma}{x}, \quad \gamma:=\frac{\delta_{1}-\delta_{0}}{2}, \quad x>0,
$$

and is therefore a non-constant function of the observed Bessel process $X$. Then, according to Section 2.2, solving (2.3) under $\mathbb{P}_{\pi}$ would lead to face the three-dimensional optimal stopping problem (2.14). However, Theorem 3.2 allows us to move from the measure $\mathbb{P}_{\pi}$ to $\mathbb{P}^{\infty}$ and, consequently, to solve the extended two-dimensional optimal stopping problem (3.16) for $(X, \Phi)$, that, from (3.1)-(3.2), satisfy

$$
\begin{aligned}
& d X_{t}=\frac{\delta_{0}-1}{2 X_{t}} d t+d W_{t}, \quad X_{0}=x \in(0, \infty), \\
& d \Phi_{t}=\left(\lambda+(\lambda+\alpha) \Phi_{t}\right) d t+\gamma \frac{\Phi_{t}}{X_{t}} d W_{t}, \quad \Phi_{0}=\phi \in[0, \infty) .
\end{aligned}
$$

Comparing (3.16) with [13, Eq. (4.19)], we can appreciate the fact that two optimal stopping problems share a similar structure, with the former being the result of a dimension reduction procedure, unlike the latter (see Remark 3.3 above). Let us also notice that in [13, Eq. (4.19)] the process $\Phi$ inside the integral stands for the posterior probability ratio process, which is a particular case of the generalized odds process $\Phi$ from (2.4) with $\alpha=0$, and the constant values $\lambda / c$ gets replaced by $\lambda /(c \alpha)$ in our case. Then, straightforward extensions of some technical results from [13, Sections $7,8,12]$ show that the first entry time of $(X, \Phi)$ into $D$ from (3.18), defined by

$$
\tau_{D}:=\inf \left\{t \geq 0:\left(X_{t}, \Phi_{t}\right) \in D\right\},
$$

is optimal in (3.16), as well as that the optimal boundary $x \mapsto b(x)$ is finite-valued and decreasing on $(0, \infty)$ and satisfies $\lim _{x \downarrow 0} b(x)=\infty$ and $\lim _{x \rightarrow \infty} b(x)=\lambda /(c \alpha)$.

\subsection{Free-boundary problem}

For the optimal stopping problem (3.16), associated to the strong Markov process $(X, \Phi)$ from (5.3)-(5.4), we can formulate the following free-boundary problem for finding $\hat{V}$ and $b$ (see, e.g., [16, Chapter 3 and 4] or [20, Chapter 3]):

$$
\begin{aligned}
& \left(\mathbb{L}_{(X, \Phi)} \hat{V}-\lambda \hat{V}\right)(x, \phi)=-\left(\phi-\frac{\lambda}{c \alpha}\right), \quad(x, \phi) \in C, \\
& \hat{V}(x, \phi)=0, \quad(x, \phi) \in D \text { (instantaneous stopping), } \\
& \hat{V}_{x}(x, b(x))=0, \quad \hat{V}_{\phi}(x, b(x))=0, \quad x>0 \text { (smooth fit), }
\end{aligned}
$$


Dimension reduction in the quickest detection problem with exponential penalty

where the infinitesimal generator $\mathbb{L}_{(X, \Phi)}$ from (3.20) takes the more specific form

$$
\mathbb{L}_{(X, \Phi)}=(\lambda+(\lambda+\alpha) \phi) \frac{\partial}{\partial \phi}+\frac{\delta_{0}-1}{2 x} \frac{\partial}{\partial x}+\gamma \frac{\phi}{x} \frac{\partial^{2}}{\partial \phi \partial x}+\frac{\gamma^{2}}{2} \frac{\phi^{2}}{x^{2}} \frac{\partial^{2}}{\partial \phi^{2}}+\frac{1}{2} \frac{\partial^{2}}{\partial x^{2}}
$$

and the continuation and stopping sets $C$ and $D$ from (3.17)-(3.18) are more explicitly given by

$$
C=\{(x, \phi) \in(0, \infty) \times[0, \infty): \phi<b(x)\}, \quad D=\{(x, \phi) \in(0, \infty) \times[0, \infty): \phi \geq b(x)\} .
$$

Clearly, the global condition (5.7) can be replaced by the local condition $\hat{V}(x, b(x))=0$ for $x>0$, so that the free-boundary problem needs to be considered on the closure of $C$ only (by extending $\hat{V}$ to zero on $D$, as evident from (3.18)). Let us now define by $\mathbf{A}$ the class of the pairs of functions $(F, h)$ such that:

$F$ belongs to $C^{1}\left(\overline{C_{h}}\right) \cap C^{2}\left(C_{h}\right)$ and is bounded on $(0, \infty) \times[0, \infty)$,

$h$ is continuous and decreasing on $(0, \infty)$ with $h(x) \geq \lambda /(c \alpha)$ for $x>0$,

where $C_{h}:=\{(x, \phi) \in(0, \infty) \times[0, \infty): \phi<h(x)\}$ and $\overline{C_{h}}:=\{(x, \phi) \in(0, \infty) \times[0, \infty)$ : $\phi \leq h(x)\}$. With this in mind, one can easily extend [13, Theorem 17] to show that the free-boundary problem (5.6)-(5.8) has a unique solution $(\hat{V}, b)$ in the class $\mathbf{A}$ from (5.11)-(5.12), where $\hat{V}$ and $b$ are given by (3.16) and (3.19), respectively.

\subsection{Nonlinear integral equation}

We can now characterize the optimal stopping boundary $b$ from (5.10) as the unique solution to a Fredholm integral equation; this in turn returns a triple integral representation of the value function $\hat{V}$ from (3.16) in terms of $b$. To this aim, let us denote by $p_{\alpha}(t ; y, \psi ; x, \phi)$ the density of $\left(X_{t}, \Phi_{t}\right)$ at $(y, \psi)$, starting at $(x, \phi)$ under $\mathbb{P}^{\infty}$ :

$$
\mathbb{P}_{x, \phi}^{\infty}\left(X_{t} \leq y, \Phi_{t} \leq \psi\right)=\int_{0}^{y} \int_{0}^{\psi} p_{\alpha}(t ; u, v ; x, \phi) d v d u
$$

with $(y, \psi)$ and $(x, \phi)$ in $(0, \infty) \times[0, \infty)$ and $t>0$. The subscript $\alpha$ to the density $p$ highlights the dependence of latter on the discounting factor $\alpha>0$ from (2.3) and appearing in (3.2) and (5.4). According to the Kolmogorov backward equation, the density $p_{\alpha}$ is the unique non-negative solution to

$$
\begin{gathered}
\frac{\partial}{\partial t} p_{\alpha}(t ; y, \psi ; x, \phi)=\left(\mathbb{L}_{(X, \Phi)} p_{\alpha}\right)(t ; y, \psi ; x, \phi), \\
p_{\alpha}(0+; y, \psi ; x, \phi)=\delta_{(x, \phi)}(y, \psi) \quad \text { (weakly), }
\end{gathered}
$$

satisfying $\int_{0}^{\infty} \int_{0}^{\infty} p_{\alpha}(t ; y, \psi ; x, \phi) d \psi d y=1$ for $t>0$ and $(y, \psi)$ and $(x, \phi)$ in $(0, \infty) \times[0, \infty)$. We recall that $\mathbb{L}_{(X, \Phi)}$ is given in (5.9) and $\delta_{(x, \phi)}$ is the Dirac measure at $(x, \phi)$. Then, (5.14)-(5.15) can be used to compute $p_{\alpha}$ in the theorem below.

Theorem 5.1. Within the class of functions complying with (5.12), the optimal stopping boundary $b$ from (5.10) is the unique solution to

$$
\int_{0}^{\infty} \int_{0}^{\infty} \int_{0}^{b(y)} e^{-\lambda t}\left(\psi-\frac{\lambda}{c \alpha}\right) p_{\alpha}(t ; y, \psi ; x, b(x)) d \psi d y d t=0, \quad x>0 .
$$

The value function $\hat{V}$ from (3.16) can be expressed as

$$
\hat{V}(x, \phi)=\int_{0}^{\infty} \int_{0}^{\infty} \int_{0}^{b(y)} e^{-\lambda t}\left(\psi-\frac{\lambda}{c \alpha}\right) p_{\alpha}(t ; y, \psi ; x, \phi) d \psi d y d t
$$


Dimension reduction in the quickest detection problem with exponential penalty

with $(x, \phi) \in(0, \infty) \times[0, \infty)$. Moreover, the optimal stopping time in the problem (3.16) is given by

$$
\tau^{\star}=\inf \left\{t \geq 0: \Phi_{t} \geq b\left(X_{t}\right)\right\}
$$

under $\mathbb{P}_{x, \phi}^{\infty}$, with $(x, \phi) \in(0, \infty) \times[0, \infty)$ given and fixed.

Proof. The proof requires minor modifications to the proof in [13, Theorem 19]; this is due to the the similar structure between the optimal stopping problems (3.16) and [13, Eq. (4.19)] obtained by means of Theorem 3.2.

Now, observing that $p_{\alpha}$ and $b$ satisfying (5.14)-(5.15) and (5.16) can be computed numerically, once they are obtained, they can be plugged in (5.17) to compute the value function $\hat{V}$. It follows that the explicit solution to the initial problem (2.3) and (2.12) for a given starting point $x>0$ of the Bessel process $X$ is given by

$$
V(\pi)=(1-\pi)\left(1+c \alpha \hat{V}\left(x, \frac{\pi}{1-\pi}\right)\right), \quad \pi \in[0,1),
$$

with $\hat{V}$ given by (5.17), as evident from (3.6)-(3.7) in Theorem 3.2 and the extended optimal stopping problem (3.16). Finally, the optimal stopping time in the initial problem (2.3) and (2.12) is

$$
\tau^{\star}=\inf \left\{t \geq 0:\left(\frac{X_{t}}{x}\right)^{\gamma} e^{(\alpha+\lambda) t-\beta A_{t}}\left(\frac{\pi}{1-\pi}+\lambda x^{\gamma} \int_{0}^{t} \frac{e^{-(\alpha+\lambda) s+\beta A_{s}}}{X_{s}^{\gamma}} d s\right) \geq b\left(X_{t}\right)\right\},
$$

where $b$ is the unique solution to (5.16) in the class of functions satisfying (5.12), with $\beta:=\left(\delta_{1}-\delta_{0}\right)\left(\delta_{1}+\delta_{0}-4\right) / 8$ and $A_{t}:=\int_{0}^{t} \frac{1}{X_{s}^{2}} d s$ for $t \geq 0$. Expressions (5.20) follows from inserting (5.1) into (2.5), the application of Itô's formula to $\gamma \log X_{t}$, which leads to

$$
L_{t}=\left(\frac{X_{t}}{x}\right)^{\gamma} e^{-\beta A_{t}}
$$

and the combination of (2.6) with (5.18) and (5.21).

\section{References}

[1] Abramowitz, M. and Stegun, I. A. (1964). Handbook of Mathematical Functions with Formulas, Graphs, and Mathematical Tables, Washington: U.S. Departmemt of Commerce. MR0208797

[2] Bayraktar, E. and Dayanik, S. (2006). Poisson disorder problem with exponential penalty for delay, Mathematics of Operations Research 31: 217-233. MR2233993

[3] Bayraktar, E., Dayanik, S., and Karatzas, I. (2005). The standard Poisson disorder problem revisited, Stochastic Processes and Their Applications 115: 1437-1450. MR2158013

[4] Beibel, M. (2000). A note on sequential detection with exponential penalty for the delay, Annals of Statistics 28: 1696-1701. MR1835037

[5] Beibel, M. and Lerche, H. R. (1997). A new look at optimal stopping problems related to mathematical finance, Statistica Sinica 7: 93-108. MR1441146

[6] Buonaguidi, B. (2020). The disorder problem for purely jump Lévy processes with completely monotone jumps, Journal of Statistical Planning and Inference 205: 203-218. MR4011632

[7] Christensen, S. and Irle, A. (2011). A harmonic function technique for the optimal stopping of diffusions, Stochastics 83: 347-363. MR2842584

[8] Dayanik, S. and Karatzas I. (2003). On the optimal stopping problem for one-dimensional diffusions, Stochastic Processes and Their Applications 107: 173-212. MR1999788

[9] Dayanik, S. and Sezer, S. O. (2006). Compound Poisson disorder problem, Mathematics of Operations Research 31: 649-672. MR2281222 
Dimension reduction in the quickest detection problem with exponential penalty

[10] Gapeev, P. V. and Peskir, G. (2006). The Wiener disorder problem with finite horizon, Stochastic Processes and Their Applications 116: 1770-1791. MR2307058

[11] Gapeev, P. V. and Shiryaev, A. N. (2013). Bayesian quickest detection problems for some diffusion processes, Advances in Applied Probability 45: 164-185. MR3077545

[12] Gapeev, P. V. and Stoev, Y. I. (2017). On the sequential testing and quickest change-point detection problems for Gaussian processes, Stochastics 89: 1143-1165. MR3742326

[13] Johnson, P. and Peskir, G. (2017). Quickest detection problems for Bessel processes, Annals of Applied Probability 27: 1003-1056. MR3655860

[14] Oksendal, B. (1998). Stochastic Differential Equations, Berlin: Springer-Verlag. MR1619188

[15] Peskir, G. (2019). Continuity of the optimal stopping boundary for two-dimensional diffusions, Annals of Applied probability 29: 505-530. MR3910010

[16] Peskir, G. and Shiryaev, A. N. (2006). Optimal Stopping and Free-Boundary Problems, Lectures in Mathematics ETH Zürich, Basel: Birkhäuser Verlag. MR2256030

[17] Polyanin, A. D. and Zaitsev, V. F. (1995). Handbook of Exact Solutions for Ordinary Differential Equations, Crc Press. MR1396087

[18] Poor, H. V. (1998). Quickest detection with exponential penalty for delay, Annals of Statistics 26: 2179-2205. MR1700227

[19] Salminen, P. (1985). Optimal stopping of one-dimensional diffusions, Mathematische Nachrichten 124: 85-101. MR0827892

[20] Shiryaev, A. N. (1978). Optimal Stopping Rules, New York: Springer-Verlag. MR0468067

Acknowledgments. The author wishes to thank the Editor and an anonymous referee for their insightful comments, which improved the presentation of the paper. 